\title{
Cost-Efficient Modeling of Antenna Structures Using Gradient-Enhanced Kriging
}

\author{
Selvakumar Ulaganathan \\ Department of Information Technology \\ Ghent University \\ Ghent, Belgium \\ selvakumar.ulaganathan@ugent.be
}

\author{
Slawomir Koziel, \\ Adrian Bekasiewicz \\ Engineering Opt. \& Modeling Center \\ Reykjavik University \\ Reykjavik, Iceland \\ \{koziel,bekasiewicz\}@ru.is
}

\author{
Ivo Couckuyt, Eric Laermans, \\ Tom Dhaene \\ Department of Information Technology \\ Ghent University, Ghent, Belgium \\ \{ivo.couckuyt,eric.laermans, \\ tom.dhaene\}@ugent.be
}

\begin{abstract}
Reliable yet fast surrogate models are indispensable in the design of contemporary antenna structures. Data-driven models, e.g., based on Gaussian Processes or support-vector regression, offer sufficient flexibility and speed, however, their setup cost is large and grows very quickly with the dimensionality of the design space. In this paper, we propose cost-efficient modeling of antenna structures using GradientEnhanced Kriging. In our approach, the training data set contains, apart from the EM-simulation responses of the structure at hand, also derivative data at the respective training locations obtained at little extra cost using adjoint sensitivity techniques. We demonstrate that introduction of the derivative information into the model allows for considerable reduction of the model setup cost (in terms of the number of training points required) without compromising its predictive power. The Gradient-Enhanced Kriging technique is illustrated using a dielectric resonator antenna structure. Comparison with conventional Kriging interpolation is also provided.
\end{abstract}

Keywords-Antenna modeling; electromagnetic simulation; surrogate modeling; Kriging; Gradient-Enhanced Kriging; computeraided design

\section{INTRODUCTION}

Full-wave electromagnetic (EM) analysis allows for accurate evaluation of antenna structures as well as for taking into account various environmental effects such as the presence of connectors, housing, installation fixtures, etc. EM simulation tools are therefore major design tools of contemporary antenna engineering. However, accurate EM analysis at fine discretization of the structure at hand is computationally expensive. Consequently, carrying out design tasks that require numerous analyses, such as parametric optimization, yield-driven design or statistical analysis, may be impractical or even prohibitive. In all these situations, fast replacement models (surrogates) become indispensable.

Implementation of low-cost antenna models is possible using various approximation techniques such as polynomial regression [1], radial basis function interpolation [2], Kriging [3], [4] support vector regression [5]-[8], fuzzy systems [9], [10] multidimensional Cauchy approximation [11], or artificial neural networks [12]-[15]. A common problem related to all of these methods is high model setup cost: in order to ensure usable accuracy a large number of training points is necessary, which quickly grows with the dimensionality of the design space (a problem referred to as the curse of dimensionality) [1], [16]. As a result, approximation techniques are mostly suitable for creating multiple-use library models but not so much to build surrogates for, say, one-time optimization of a specific antenna structure.

Physics-based surrogate modeling is another way of creating fast and of usable-accuracy replacement models. Perhaps the most popular type of technique of this kind is space mapping (SM) [17]-[20], where the surrogate is constructed by means of suitable correction of an underlying low-fidelity (or so-called coarse) model. The bottleneck of space mapping in terms of antenna modeling is the lack of fast coarse models, because low-fidelity antenna representations are normally obtained through coarse-discretization EM simulations, the cost of which cannot be neglected. Another issue with SM is fixed number of extractable parameters which limits the model flexibility. This particular difficulty can be alleviated, to some extent by SM enhancement through fuzzy systems [21], radial-basis functions [18], or Kriging [22].

The problem of excessive number of training samples necessary to establish a reliable surrogate can be partially addressed by modeling methods that rely on appropriately extracted response features (e.g., shape-preserving response prediction [23], or feature-based modeling [24]), however, these methods impose relatively strong assumptions on the response shapes of the structures under consideration so their applicability is limited to certain types of devices [24]. Another possibility is variable-fidelity modeling, where densely sampled coarse-mesh and sparsely-sampled fine-mesh simulation data are blended together using, e.g., co-Kriging [25], [34] or space mapping [35], [36]. An interesting variable-fidelity Gaussian Process regression modeling procedure has been reported in [26].

In this paper, we propose low-cost modeling of antenna structures using Gradient-Enhanced Kriging. The cost reduction is achieved by exploiting cheap derivative information obtained using adjoint sensitivities (currently, 
available through certain commercial EM solvers such as CST [27] or HFSS [28]). We demonstrate that exploiting response gradients in the Kriging model leads to significant reduction of the number of training data without degradation of the predictive power of the model. Our approach is illustrated using a dielectric resonator antenna and compared to conventional Kriging interpolation that utilized the response data only.

\section{KRIGING AND GRADIENT-ENHANCED KRIGING BASICS}

In this section, we briefly outline the formulation of Kriging and Gradient-Enhanced Kriging (GEK) interpolation in the context of antenna modeling.

\section{A. Surrogate Modeling of Antenna Structures}

Let $\boldsymbol{f}(\boldsymbol{x})$ be an EM-simulated antenna model, where $\boldsymbol{x}$ is a vector of geometry parameters, whereas $f$ is a vector-valued response, e.g., reflection coefficient versus frequency. In practice, we assume that $\boldsymbol{f}(\boldsymbol{x})=\left[f\left(\boldsymbol{x}, \omega_{1}\right) f\left(\boldsymbol{x}, \omega_{2}\right) \ldots f\left(\boldsymbol{x}, \omega_{m}\right)\right]^{T}$, i.e., the model is evaluated at a discrete set of frequencies $\omega_{k}$, $k=1, \ldots, m$. The EM model is assumed to be computationally expensive. The goal of the surrogate modeling process is to construct a cheaper representation $s$ of $f$ that is valid in a certain domain, usually an interval $\boldsymbol{l} \leq \boldsymbol{x} \leq \boldsymbol{u}$, where $\boldsymbol{l}$ and $\boldsymbol{u}$ are the lower and upper bounds for the geometry parameters, respectively.

\section{B. Gradient-Enhanced Kriging}

Ordinary Kriging (OK) prediction of $\boldsymbol{f}(\boldsymbol{x})$ at a prediction (or untried) point $\boldsymbol{x}^{*}$ can be expressed as a summation of a constant trend function $\mu$ and a realization of a stationary Gaussian random process:

$$
\hat{y}\left(\boldsymbol{x}^{*}\right)=\hat{\mu}+\psi^{T} \Psi^{-1} \cdot(\boldsymbol{y}-\boldsymbol{t} \hat{\mu}),
$$

where $\psi$ is a vector of correlations between $\mathrm{n}_{\mathrm{s}}$ sample data points $X=\left\{\boldsymbol{x}^{(1)}, \ldots, \boldsymbol{x}^{\left(n_{s}\right)}\right\}^{T}$ and prediction point $\boldsymbol{x} ; \Psi$ is a $n_{s} \times$ $n_{s}$ symmetric matrix of correlations between the sample data points; $\boldsymbol{y}$ is a vector of response values and $\boldsymbol{t}$ is a vector of ones. The trend function is calculated using least squares method as,

$$
\hat{\mu}=\left(\boldsymbol{I}^{T} \Psi^{-1} \boldsymbol{I}\right)^{-1} \boldsymbol{I}^{T} \Psi^{-1} \boldsymbol{y} .
$$

The covariance structure of the sample data is captured using Matern 3/2 correlation function [29]-[30] which is expressed as

$$
\psi\left(d^{\prime}\right)=(1+\sqrt{3} c) \exp (-\sqrt{3} c)
$$

where $c=\left(\sum_{p}=1, \ldots, k \theta_{p} d_{p}^{\prime 2}\right)^{1 / 2}, d^{\prime}=\left|x_{p}{ }^{i}-x_{p}{ }^{j}\right|$ and $k$ is the dimensionality of the problem. The maximum likelihood estimate of the hyper-parameters of the correlation function is estimated by maximizing the concentrated $\ln$-likelihood function

$$
\phi=\left[-n_{s} \ln \left(\hat{\sigma}^{2}\right)-\ln |\Psi|\right] / 2,
$$

where

$$
\hat{\sigma}^{2}=(\boldsymbol{y}-\boldsymbol{t} \hat{\mu})^{T} \Psi^{-1}(\boldsymbol{y}-\boldsymbol{t} \boldsymbol{\mu}) / n_{s}
$$

is the estimated Kriging variance.

Gradient-Enhanced Kriging is considered as a multi-data extension to Kriging. GEK incorporates cheaply available gradient data in addition to response data in building surrogate models. The mathematical formulation of GEK is same as Kriging except the following facts:

- Correlation matrix in GEK becomes a $\left(k^{\prime}+1\right) n_{s} \times\left(k^{\prime}+1\right) n_{s}$ symmetric block matrix and now contains the correlations of both response and gradient observations between the sample data points;

- Correlation vector $\psi$ now contains correlation of both response and gradient observations between the sample data points and the prediction point;

- Vector $\boldsymbol{y}$ now contains both the response and gradient values;

- Vector $\boldsymbol{t}$ now contains $n_{s}$ ones followed by $k^{\prime} \times n_{s}$ additional zeros.

More information on the mathematical formulations of Kriging and GEK can be found in [31].

\section{CASE STUdy AND Numerical Results}

In this section we present the case-study-based verification of the Gradient-Enhanced Kriging modeling technique described in Section II. We use an example of a dielectric resonator antenna. The computational cost of the model construction using GEK is compared with conventional approach that does not use the derivative data.

\section{A. Suspended Dielectric Resonator Antenna}

Consider a suspended dielectric resonator antenna (DRA) shown in Fig. 1 [32]. The structure is composed of a dielectric resonator $\left(\varepsilon_{r}=10\right.$ and $\left.\tan \delta=0.0001\right)$ suspended over the ground plane on the two Teflon slabs with relative permittivity and loss tangent of 2.1 and 0.001 , respectively (see Fig. 1(b)(c)). The antenna is fed through the ground plane slot. The substrate material is Rogers RO4003 $\left(\varepsilon_{r}=3.38, \tan \delta=0.0027\right.$ and $h=0.5 \mathrm{~mm})$. The DRA is covered by polycarbonate housing $\left(\varepsilon_{r}=2.8\right.$ and $\left.\tan \delta=0.01\right)$.

The antenna is described by a set of five independent design variables: $\boldsymbol{x}=\left[\begin{array}{lllll}a_{c} & u_{s} & w_{s} & y_{s} & g_{1}\end{array}\right]^{T}$, whereas the dimensions $d_{x}=d_{y}=d_{z}=1, a_{x}=8.06, a_{y}=14.24, a_{z}=9.25, b_{y}=5.87, d_{z b}=$ $2, b_{x}=2$, and $c_{x}=6.5$ remain fixed. The unit for all parameters is $\mathrm{mm}$. The EM model of the structure is implemented in HFSS and simulated using its frequency domain solver [28]. It consists of about 8,500 tetrahedral mesh cells and its average evaluation time is $10 \mathrm{~min}$. The design variable space for the antenna parameters is defined by the following lower and upper bounds: $\boldsymbol{l}=\left[\begin{array}{lllll}-0.5 & 1.4 & 8 & 2.4 & 1.2\end{array}\right]^{T}$ and $\boldsymbol{u}=\left[\begin{array}{llll}0.5 & 2.1 & 12 & 3.6\end{array}\right.$ $1.8]^{T}$.

\section{B. Error Metrics}

Two error metrics, Normalized Root Mean Square Error (NRMSE) and Relative Average Absolute Error (RAAE), are used to assess the accuracy of the surrogate models on a validation data set of $n_{p}$ uniformly distributed pseudorandom points. The error metrics are expressed as 


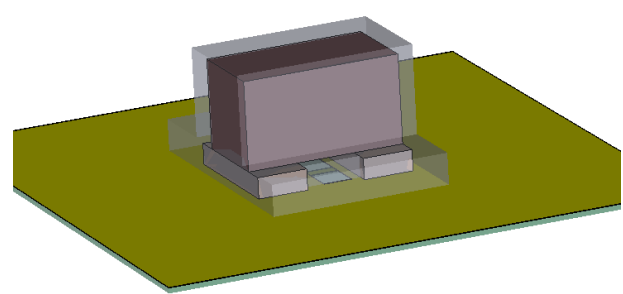

(a)

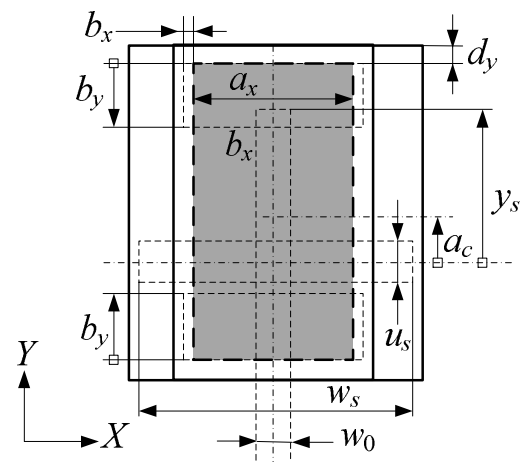

(b)

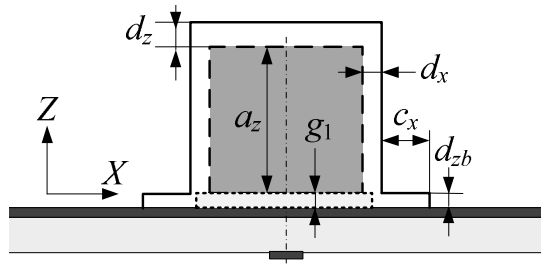

(c)

Fig. 1. The suspended dielectric resonator antenna: (a) 3D design visualization; (b) top view; (c) front view.

$$
N R M S E=\sqrt{n_{p}^{-1} \sum_{i=1}^{n_{p}}\left(y_{t}^{i}-\hat{y}^{i}\right)^{2}} /\left[\max \left(\boldsymbol{y}_{t}\right)-\min \left(\boldsymbol{y}_{t}\right)\right]
$$

and

$$
R A A E=\left(\sum_{i=1}^{n_{p}}\left|y_{t}^{i}-\hat{y}^{i}\right|\right) /\left[n_{p} \operatorname{std}\left(\boldsymbol{y}_{t}\right)\right]
$$

where $\boldsymbol{y}_{t}$ is the vector of true response values, $\hat{\boldsymbol{y}}$ is the vector of predicted response values and std stands for standard deviation. NRMSE and RAAE show the overall surrogate modeling accuracy and their values approach zero as the overall surrogate model accuracy increases.

\section{Numerical Results}

Figures 2 through 5 compare the actual reflection characteristic $\left|S_{11}\right|$ versus frequency to its OK and GEK approximations. GEK approximates the actual $\mid S_{11} I$ curve more accurately than $\mathrm{OK}$ while requiring only half (or even less) of the training data demanded by its counterpart (Fig. 2 and Table I). This is essentially due to the incorporation of gradient data in GEK. Gradient data in GEK not only acts as an additional data but also enables GEK to successfully capture the sample data's covariance structure by forcing GEK to interpolate response and gradient data [33]. Moreover, it is worth noting that the OK approximation with $n_{s}=100$ does not overlay the GEK approximation with $n_{s}=20$, thus indicating the fact that GEK achieves an accuracy level which is almost never reached by $\mathrm{OK}$ for any given number of training sample points. The same can be perceived in Fig. 6 which depicts the evolution of NRMSE as a function of number of training sample points.

Table II showcases the efficiency of GEK by presenting its rate of accuracy improvement over OK over a range of number of training sample points. It can be observed that GEK is more effective when the training data set is smaller and, in this case, achieves more than $50 \%$ of accuracy improvement over OK. However, as the amount of training data increases, the advantage of incorporating additional gradient data gradually thins (reduces to $10 \%-20 \%$ of accuracy improvement in this case). The effect of incorporating partial set of gradients in Fig. 7 confirms the same conjecture. A reasonable percentage of accuracy improvement is observed when the additional gradient data in GEK is incorporated only in three geometrical dimensions $\left(a_{c} u_{s} w_{s}\right)$.

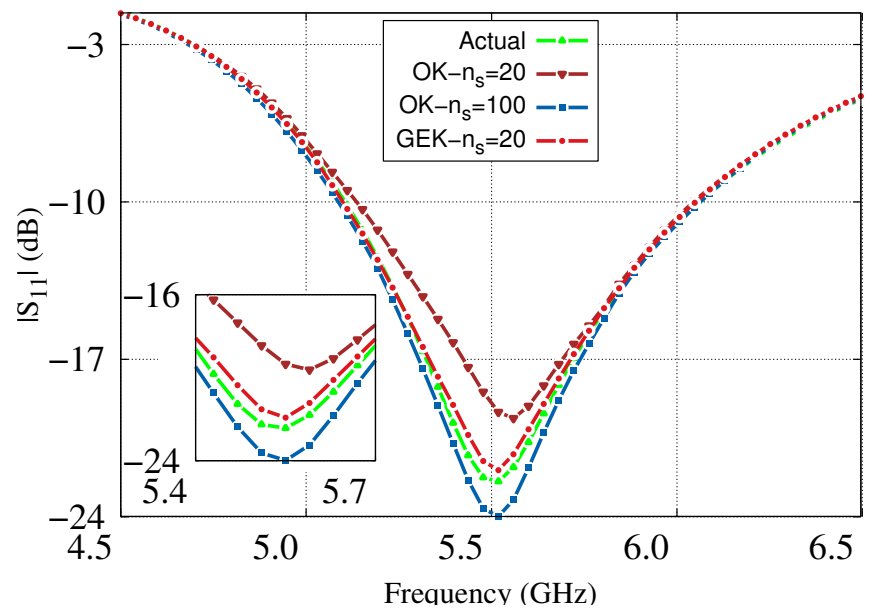

Fig. 2. Modeling of $f(x)$ at the test point 1 . OK incorporates only the response data whereas GEK incorporates gradient data in addition to the response data.

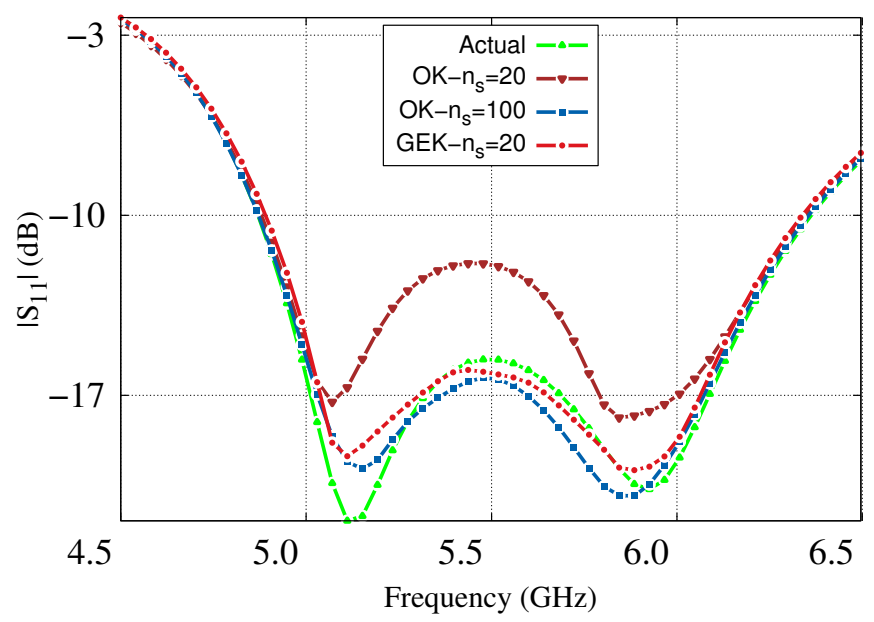

Fig. 3. Modeling of $\boldsymbol{f}(\boldsymbol{x})$ at the test point 2. OK incorporates only the response data whereas GEK incorporates gradient data in addition to the response data. 


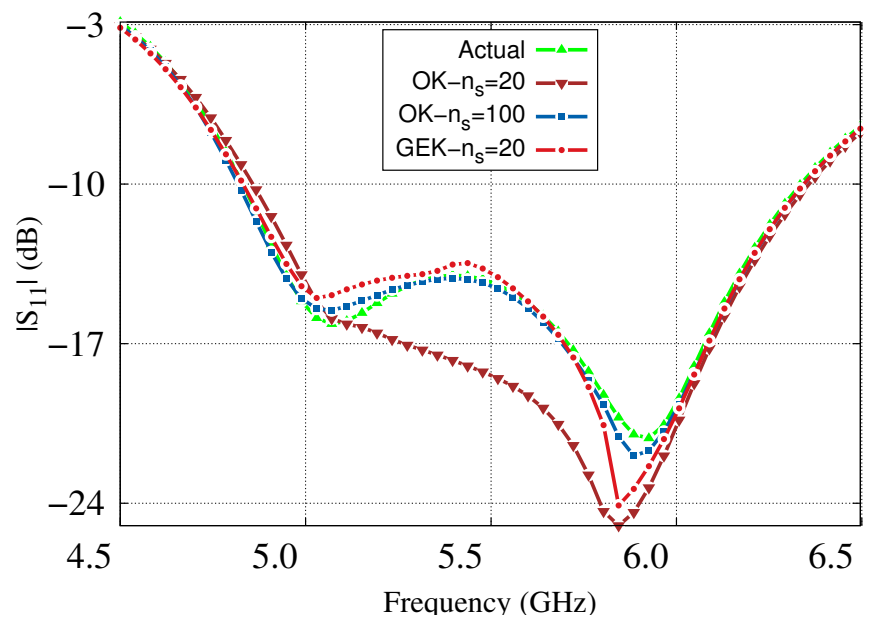

Fig. 4. Modeling of $\boldsymbol{f}(\boldsymbol{x})$ at the test point 3. OK incorporates only the response data whereas GEK incorporates gradient data in addition to the response data.

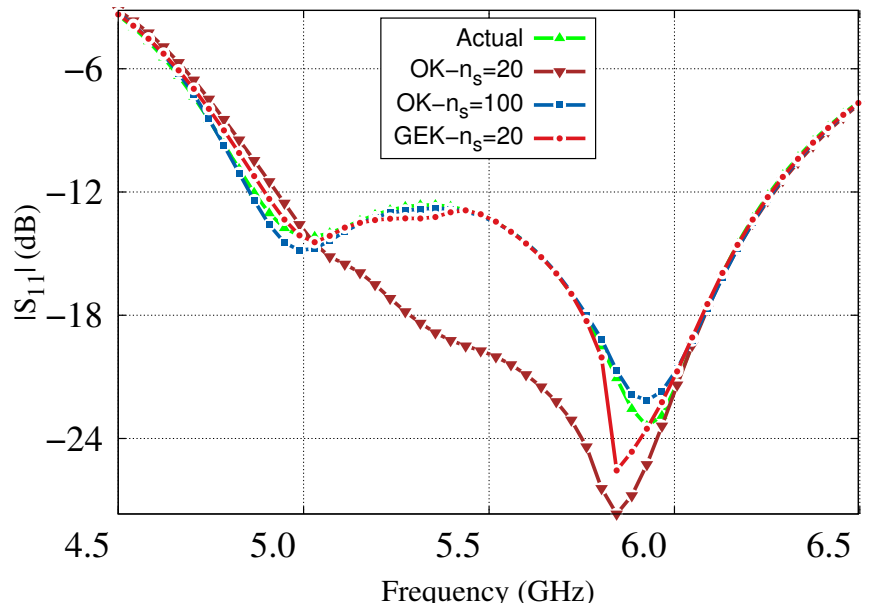

Fig. 5. Modeling of $\boldsymbol{f}(\boldsymbol{x})$ at the test point 4 . OK incorporates only the response data whereas GEK incorporates gradient data in addition to the response data.

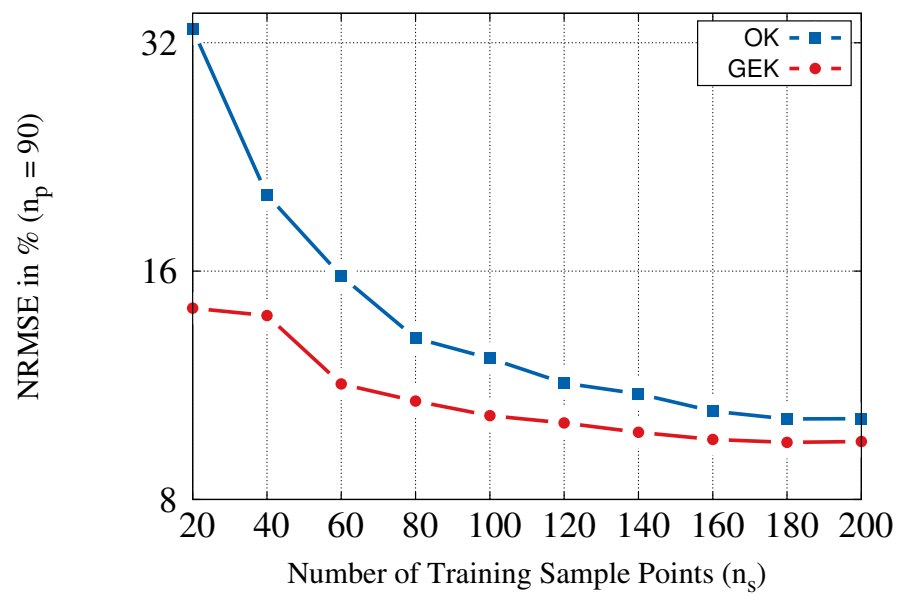

Fig. 6. Evolution of NRMSE on a validation data set of $n_{p}=90$ for a varying number of training sample points. OK incorporates only the response data wheres GEK incorporates gradient data in addition to the response data.
However, the advantage of incorporating additional gradient data in GEK is overshadowed by the incorporation of more response data in OK (at and after 120 samples in Fig. 7). This confirms the intuitive fact that a response value is worth more than a gradient value. Although acquiring more response data often comes at a greater computational cost than that of acquiring gradient data, this rather reminds one the value of response information.

TABLE I. REDUCTION IN NUMBER OF TRAINING SAMPLES WITH GRADIENT ENHANCED KRIGING*

\begin{tabular}{|c|c|c|c|}
\hline Model & $\begin{array}{c}\text { Number of } \\
\text { Training Samples }\end{array}$ & $\begin{array}{c}\text { NRMSE } \\
(\boldsymbol{\%})\end{array}$ & $\begin{array}{c}\text { Percentage of } \\
\text { Reduction in } \\
\text { Training Samples }\end{array}$ \\
\hline OK & 200 & 10.2 & -- \\
GEK & 60 & 11.3 & $70 \%$ \\
GEK & 80 & 10.7 & $60 \%$ \\
GEK & 100 & 10.3 & $50 \%$ \\
\hline
\end{tabular}

"Based on normalized root mean square error (NRMSE) on a validation data set of 90 points.

TABLE II. EFFICIENCY OF GEK OVER OK BASED ON RELATIVE AVERAGE ABSOLUTE ERROR (RAAE) MEASURE

\begin{tabular}{|c|c|c|c|c|}
\hline \multirow{2}{*}{$\begin{array}{c}\text { Number of } \\
\text { Training } \\
\text { Samples }\end{array}$} & \begin{tabular}{c} 
RAAE \\
\cline { 2 - 5 }
\end{tabular} & $\begin{array}{c}\text { OK } \\
\text { Percentage of } \\
\text { Accuracy } \\
\text { Improvement } \\
\text { of OK }\end{array}$ & $\begin{array}{c}\text { RAAE } \\
(\%)\end{array}$ & $\begin{array}{c}\text { Percentage of } \\
\text { Accuracy } \\
\text { Improvement } \\
\text { of GEK over } \\
\text { OK }\end{array}$ \\
\hline 20 & 13.2 & -- & 5.9 & $55 \%$ \\
40 & 8.4 & $37 \%$ & 5.8 & $56 \%$ \\
60 & 6.8 & $48 \%$ & 4.8 & $64 \%$ \\
80 & 5.9 & $55 \%$ & 4.7 & $65 \%$ \\
100 & 5.6 & $57 \%$ & 4.5 & $66 \%$ \\
\hline
\end{tabular}

* The value of RAAE which corresponds to OK model at $n_{p}=20$ is used as a base value to calculate the rate of accuracy improvement.

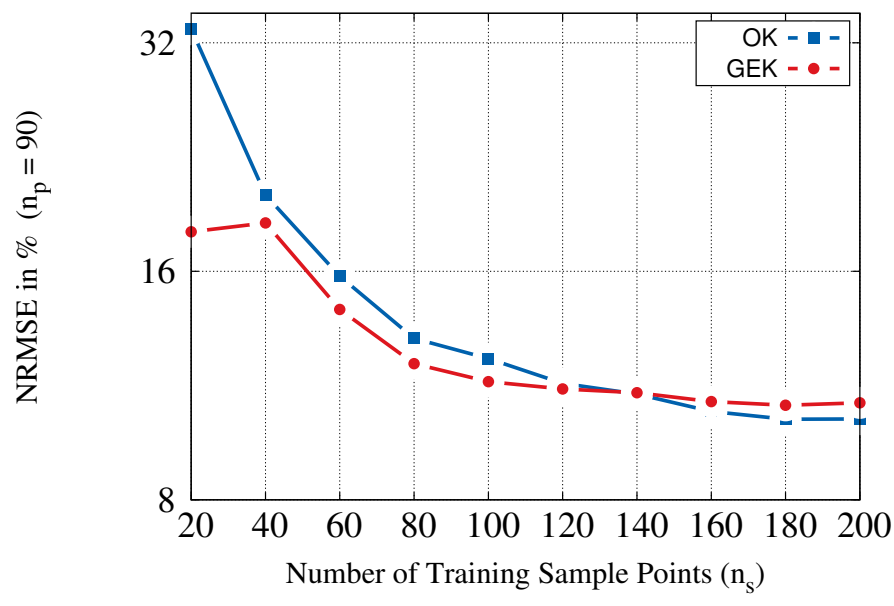

Fig. 7. Evolution of NRMSE with partial set of gradients. GEK incorporates additional gradient data in the direction of three geometric variables only. The validation data set contains 90 untried points. 


\section{CONCLUSION}

In this work, a cost-efficient surrogate modeling of antenna structures using Gradient-Enhanced Kriging has been demonstrated. As shown on the example of the suspended DRA, utilization of the sensitivity data considerably reduces the number of the training samples required to set up a fast and reliable antenna model, compared to the conventional approach that only relies on the response data. Computational savings are possible by taking advantage of adjoint sensitivities that allow for obtaining the response gradients at little extra CPU cost.

\section{ACKNOWLEDGMENT}

This research has been funded in part by the Interuniversity Attraction Poles Programme BESTCOM initiated by the Belgian Science Policy Office. Additionally, it has been supported by the Fund for Scientific Research in Flanders (FWO-Vlaanderen). Ivo Couckuyt is a post-doctoral research fellow of the Research Foundation Flanders (FWOVlaanderen).

\section{REFERENCES}

[1] A.I.J. Forrester and A.J. Keane, "Recent advances in surrogate-based optimization," Prog. Aerospace Sci., vol. 45, no. 1-3, pp. 50-79, Jan.April, 2009

[2] M.D. Buhmann and M.J. Ablowitz, Radial Basis Functions: Theory and Implementations, Cambridge University, 2003.

[3] I. Couckuyt, T. Dhaene, and P. Demeester, "ooDACE toolbox: A flexible object-oriented kriging implementation," J. Machine Learning Research, vol. 15, pp. 3183-3186, 2014.

[4] D. Gorissen, I. Couckuyt, P. Demeester, T. Dhaene, and K. Crombecq, "A surrogate modeling and adaptive sampling toolbox for computer based design," J. Machine Learning Research, vol. 11, pp. 2051-2055, 2010.

[5] A.J. Smola and B. Schölkopf, "A tutorial on support vector regression," Statistics and Computing, vol. 14, no. 3, pp. 199-222, Aug. 2004.

[6] J. Meng and L. Xia, "Support-vector regression model for millimeter wave transition," Int. J. Infrared and Milimeter Waves, vol. 28, no. 5, pp. 413-421, May 2007.

[7] L. Xia, J. Meng, R. Xu, B. Yan, and Y. Guo, "Modeling of 3-D vertical interconnect using support vector machine regression," IEEE Microwave Wireless Comp. Lett., vol. 16, no. 12, pp. 639-641, Dec. 2006.

[8] M. Martinez-Ramon and C. Christodoulou, "Support vector machines for antenna array processing and electromagnetics," Synthesis Lectures on Computational Electromagnetics, vol. 1, no. 1, 2006.

[9] V. Miraftab and R.R. Mansour, "EM-based microwave circuit design using fuzzy logic techniques" IEE Microwaves, Ant. Prop., vol. 153, no. 6, pp. 495-501, Dec. 2006.

[10] J. Zhai, J. Zhou, L. Zhang, and W. Hong, "Behavioral modeling of power amplifiers with dynamic fuzzy neural networks," IEEE Microwave Wireless Comp. Lett., vol. 20, no. 9, pp. 528-530, 2010.

[11] G.S.A. Shaker, M.H. Bakr, N. Sangary, and S. Safavi-Naeini, "Accelerated antenna design methodology exploiting parameterized Cauchy models," PIER-99, pp. 279-309, 2009.

[12] J.E. Rayas-Sánchez, "EM-based optimization of microwave circuits using artificial neural networks: the state-of-the-art," IEEE Trans. Microwave Theory Tech., vol. 52, no. 1, pp. 420-435, Jan. 2004.

[13] H. Kabir, Y. Wang, M. Yu, and Q.J. Zhang, "Neural network inverse modeling and applications to microwave filter design," IEEE Trans. Microwave Theory Tech., vol. 56, no. 4, , pp. 867-879, April 2008.
[14] Y. Cao, X. Chen, and G. Wang, "Dynamic behavioral modeling of nonlinear microwave devices using real-time recurrent neural network," IEEE Trans. Electron Devices, vol. 56, no. 5, pp. 1020-1026, 2009.

[15] H. Kabir, Y. Wang, M. Yu, and Q.J. Zhang, "High-dimensional neuralnetwork technique and applications to microwave filter modeling," IEEE Trans. Microwave Theory Tech., vol. 58, no. 1, pp. 145-156, Jan. 2010.

[16] S. Koziel, A. Bekasiewicz, and W. Zieniutycz, "Expedite EM-Driven Multi-Objective Antenna Design in Highly-Dimensional Parameter Spaces," IEEE Ant. Wireless Propag. Lett., vol. 13, pp. 631-634, 2014.

[17] E. Rayas-Sánchez and V. Gutierrez-Ayala, "EM-based Monte Carlo analysis and yield prediction of microwave circuits using linear-input neural-output space mapping," IEEE Trans. Microwave Theory Tech., vol. 54, no. 12, pp. 4528-4537, 2006.

[18] S. Koziel and J.W. Bandler, "Recent advances in space-mapping-based modeling of microwave devices," Int. J. Numerical Modelling, vol. 23, no. 6, pp. 425-446, 2010.

[19] L. Zhang, Q.J. Zhang, and J. Wood, "Statistical neuro-space mapping technique for large-signal modeling of nonlinear devices," IEEE Trans. Microwave Theory Tech., vol, 56, no. 1, pp. 2453-2467, 2011.

[20] J.W. Bandler, Q.S. Cheng, and S. Koziel, "Simplified space mapping approach to enhancement of microwave device models," Int. J. RF and Microwave Computer-Aided Eng., vol. 16, no. 5, pp. 518-535, 2006.

[21] S. Koziel and J.W. Bandler, "A space-mapping approach to microwave device modeling exploiting fuzzy systems," IEEE Trans. Microwave Theory Tech., vol. 55, no. 12, pp. 2539-2547, Dec. 2007.

[22] S. Koziel and J.W. Bandler, "Accurate modeling of microwave devices using space mapping and Kriging," Int. Review Prog. Applied Comp. Electromagnetics, pp. 902-907, 2010.

[23] S. Koziel, Q.S. Cheng, and J.W. Bandler, "Fast EM modeling exploiting shape-preserving response prediction and space mapping," to appear, IEEE Trans. Microwave Theory Tech., 2014.

[24] S. Koziel and J.W. Bandler, "Rapid yield estimation and optimization of microwave structures exploiting feature-based statistical analysis," IEEE Trans. Microwave Theory Tech., vol. 63, no., 1, pp. 107-114, 2015.

[25] S. Koziel, S. Ogurtsov, I. Couckuyt, and T. Dhaene, "Variable-Fidelity Electromagnetic Simulations and Co-Kriging for Accurate Modeling of Antennas," IEEE Trans. Ant. Prop., vol. 61, no. 3, pp. 1301-1308, 2013.

[26] J.P. Jacobs and S. Koziel, "Two-stage framework for efficient Gaussian process modeling of antenna input characteristics," IEEE Trans. Antennas Prop., vol. 62, no. 2, pp. 706-713, 2014.

[27] CST Microwave Studio, ver. 2013, CST AG, Bad Nauheimer Str. 19, D64289 Darmstadt, Germany, http://www.cst.com, 2013.

[28] Ansys HFSS, ver. 14.0 (2012), ANSYS, Inc., Southpointe 275 Technology Drive, Canonsburg, PA 15317.

[29] C. E. Rasmussen and C. K. I. Williams, "Gaussian processes for machine learning", The MIT Press, Cambridge, MA, USA, 2006.

[30] M. L. Stein, "Interpolation of Spatial Data: Some Theory for Kriging", Springer, New York, 1999.

[31] S. Ulaganathan, I. Couckuyt, T. Dhaene, J. Degroote and E. Laermans, "Performance study of gradient enhanced kriging", Engineering with computers, DOI: 10.1007/s00366-015-0397-y, 2014.

[32] S. Koziel, A. Bekasiewicz, "Variable-fidelity optimization of antennas using adjoint sensitivities," Loughborough Ant. Prop. Conf., pp. 412-415, 2014.

[33] S. Ulaganathan, I. Couckuyt, F. Ferranti, E. Laermans and T. Dhaene, "Performance study of multi-fidelity gradient enhanced kriging", Structural and Multidisciplinary Optimization, DOI: 10.1007/s00158014-1192-x, 2014.

[34] S. Koziel, A. Bekasiewicz, I. Couckuyt, and T. Dhaene, "Efficient multiobjective simulation-driven antenna design using co-kriging," IEEE Trans. Antennas Prop., vol. 62, no. 11, pp. 5900-5905, 2014.

[35] S. Koziel and A. Bekasiewicz, "Fast multi-objective optimization of narrow-band antennas using RSA models and design space reduction," IEEE Antennas and Wireless Prop. Lett., vol. 14, pp. 450-453, 2015.

[36] S. Koziel, and A. Bekasiewicz, "Strategies for computationally feasible multi-objective simulation-driven design of compact RF/microwave components," Eng. Comp., 2015. 